\title{
Evaluation de la mesure d'impédance comme technique rapide d'appréciation de la qualité bactériologique du lait cru
}

\author{
Christine PITON et A. DASEN \\ avec la collaboration technique d'Isabelle BARDOUX \\ INRA, Station expérimentale laitière, BP $n^{\circ}$ 94, 39800 Poligny, France
}

\section{Résumé}

L'impédance est une technique indirecte d'estimation de la flore totale du lait cru ; elle utilise les modifications de conductance d'un milieu de culture, en raison de la croissance et du métabolisme de la flore microbienne de l'échantillon. Il existe une relation linéaire décroissante entre le temps nécessaire pour noter une augmentation significative de la conductance (temps de détection, TD) et le niveau initial de micro-organismes dans l'échantillon $\left(\right.$ en $\left.\log _{10}\right)$.

Les échantillons de lait cru ont été analysés en double par la technique de référence (technique Spiral, SPC) et par conductance sur le Bactometer M123. Pour les mesures de conductance, nous avons évalué les conditions expérimentales suivantes : les températures d'incubation de $18^{\circ} \mathrm{C}$ et $30^{\circ} \mathrm{C}$; l'inoculation des échantillons de lait dans les cuvettes des modules en surface et dans la masse du milieu gélosé.

Deux cent quarante trois échantillons de lait contenant entre $3.10^{3}$ et $6.10^{6} \mathrm{UFC} / \mathrm{ml}$ ont été analysés par la technique de référence et par impédance à $30^{\circ} \mathrm{C}$ avec un ensemencement en surface. Le coefficient de corrélation entre les deux techniques est de $-0,657$ et l'écart-type résiduel de la référence en fonction de TD selon le modèle de régression linéaire est de 0,441 en $\log _{10} \mathrm{UFC} / \mathrm{ml}$. Cette précision s'améliore si on analyse des laits de grand mélange $\left(\mathrm{s}_{\mathrm{y}, \mathrm{x}}=0,367\right)$.

L'analyse simultanée de 150 échantillons de lait cru à $30^{\circ} \mathrm{C}$ et $18^{\circ} \mathrm{C}$ a montré que la précision d'estimation de la technique expérimentale est meilleure à $30^{\circ} \mathrm{C}$ qu'à $18^{\circ} \mathrm{C}$ $\left(\mathrm{s}_{\mathrm{y}, \mathrm{x}}=0,491\right.$ à $30^{\circ} \mathrm{C}$ contre 0,591 à $\left.18^{\circ} \mathrm{C}\right)$. Les précisions obtenues avec les deux modes d'ensemencement des échantillons sont sensiblement identiques.

Le coefficient de variation de répétabilité (en heures) est en moyenne de $5,1 \%$ avec l'inoculation en surface et de 3,6 \% avec l'inoculation dans la masse du milieu gélosé. Pour cette dernière méthode, l'écart-type de répétabilité exprimé en $\mathrm{UFC} / \mathrm{ml}$ est inférieur à la valeur limite admise pour les techniques classiques de dénombrement $\left(s_{\mathrm{r}}=0,07\right)$ et il est à peu près constant pour tous les niveaux de contamination microbienne du lait. Par contre, pour l'ensemencement en surface, l'écart-type de répétabilité augmente très nettement lorsque le niveau de contamination du lait baisse ; il dépasse $30 \%$ lorsque ce niveau est inférieur à $5.10^{4} \mathrm{UFC} / \mathrm{ml}$.

Mots clés : Lait cru - Impédance - Conductance - Dénombrement microbien - Précision Fidélité - Bactometer. 


\section{Summary}

\section{Assessment of the impedance measure for a rapid evaluation of the bacteriological quality of raw milk}

Impedance is an indirect method for the rapid evaluation of the bacteriological quality of raw milk; conductance of a growth medium is altered with chemical changes brought about by microbial metabolism and growth. Time required to bring about readily detectable change (Detection time, DT) is a linear function of the initial level of microorganisms in the samples (in $\log _{10}$ ).

Raw milk samples have been analysed in duplicate by impedance with the Bactometer M123 and the reference method (Spiral Plate Count, SPC). The following experimental conditions have been tested for the conductance measures : incubation temperatures of $30^{\circ} \mathrm{C}$ and $18^{\circ} \mathrm{C}$; running of the sample on top of the solid medium in the well (onMPCA method) and mixing of the sample with the medium (in-MPCA method).

A series of 243 raw milk samples, ranging from $3.10^{3}$ to $6.10^{6} \mathrm{CFU} / \mathrm{ml}$, have been analysed by the reference method and the impedance on-MPCA method at $30{ }^{\circ} \mathrm{C}$. The coefficient of correlation between the two methods is -0.657 and the residual standard deviation of the linear regression SPC on DT is 0.441 in $\log _{10}$ CFU $/ \mathrm{ml}$. This accuracy seems to be improved with the analysis of bulked tanker milks $\left(s_{y \cdot x}=0.367\right)$.

The analysis of 150 raw milk samples either at $30^{\circ} \mathrm{C}$ or at $18^{\circ} \mathrm{C}$ showed that the accuracy of the impedance method is better at $30^{\circ} \mathrm{C}$ than at $18{ }^{\circ} \mathrm{C}\left(\mathrm{s}_{\mathrm{y} . \mathrm{x}}=0.491\right.$ at $30^{\circ} \mathrm{C}$ and 0.591 at $18{ }^{\circ} \mathrm{C}$ ). The standard deviations from the regression obtained with the in-MPCA and the on-MPCA methods are similar.

The coefficient of variation of repeatability (in hours) averages $5.1 \%$ for the onMPCA method and $3.6 \%$ for the in-MPCA method. For this second method, the standard deviation of repeatability expressed in $\mathrm{CFU} / \mathrm{ml}$ is lower than the generally admitted value for the reference standard plate counts $\left(s_{\mathrm{r}}=0.07\right)$ and it is nearly constant at the various levels of milk contamination. But for the on-MPCA method, this standard deviation becomes proportionally higher as the level of milk contamination decreases.

Key words : Raw milk - Impedance - Conductance - Microbial count - Accuracy - Precision - Bactometer.

\section{Introduction}

L'évaluation de la qualité bactériologique du lait cru à l'aide des techniques normalisées pose souvent des problèmes à l'industriel laitier qui désire connaître rapidement la qualité de la matière première mise en œuvre. Pour répondre à ce besoin, des méthodes basées en particulier sur la mesure d'un signal lié à la présence ou à l'activité des micro-organismes ont été développées depuis une dizaine d'années (Pettipher, 1981 ; Bossuyt, 1982 a ; O’Toole, 1983 ; O'Connor, 1984). C'est le cas par exemple des variations d'impédance qui interviennent dans un milieu nutritif au cours de la croissance microbienne.

L'impédance est la résistance que subit un courant alternatif pour traverser un matériau conducteur. C'est une entité assez complexe résultant de deux propriétés du matériau : sa conductance et sa capacitance. Dans le cas de l'utilisation de 
milieux nutrififs peu tamponnés comme le Plate Count Agar, le métabolisme microbien se traduit principalement par des changements significatifs de conductance (FIRSTENBERG et ZINDULIS, 1984). Ces changements sont liés à la dégradation des substrats du milieu en molécules plus petites, plus mobiles et d'une charge électrique plus élevée comme, par exemple, celle des protéines en acides aminés ou des hydrates de carbone en lactate. Généralement, cette production d'ions devient suffisante pour entraîner une modification significative de la conductance du milieu (seuil de détection) lorsque le niveau de la population microbienne dans la cuvette de mesure a atteint $10^{6}$ à $10^{8}$ micro-organismes/ml (HADLEY et SENYK, 1975 ; Suhren et Heeschen, 1985 ; Firstenberg-Eden et Eden, 1984). Le temps (ou nombre de doublements de la population) nécessaire pour atteindre le seuil de détection sera d'autant plus grand que le niveau de contamination initial du lait sera faible (fig. 1). On peut démontrer mathématiquement et expérimentalement (FirSTENBERG-Eden et Eden, 1984) qu'il existe une relation linéaire décroissante entre le temps de détection du changement de conductance et le nombre initial de micro-organismes.

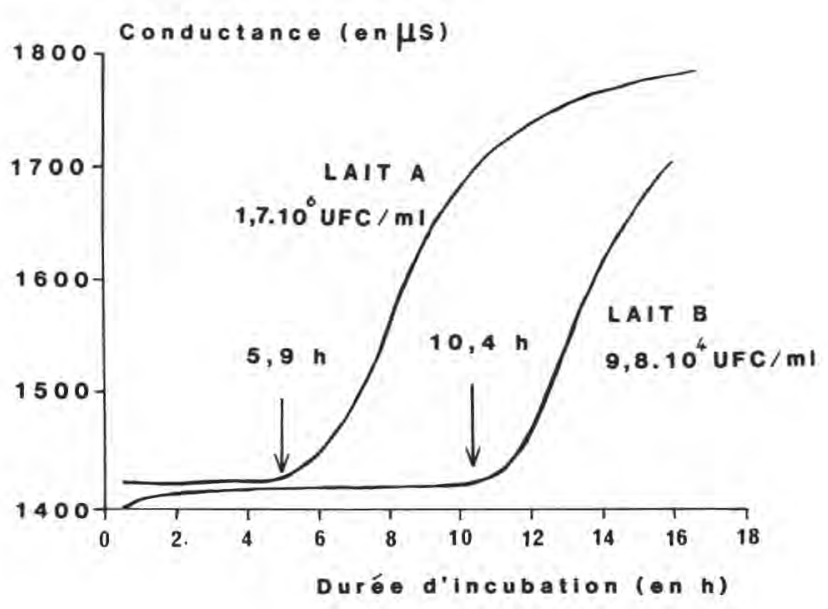

Fig. 1

Évolution de la conductance du milieu Plate Count Agar modifié au cours d'une incubation à $30^{\circ} \mathrm{C}$, pour deux échantillons de lait.

Evolution of the conductance of the Modified Plate Count Agar medium during the incubation at $30{ }^{\circ} \mathrm{C}$ of two milk samples.

L'utilisation des mesures de conductance pour l'appréciation de la qualité bactériologique du lait cru a déjà fait l'objet de nombreux travaux (CADY et al., 1978 ; O’Cr nNor, 1979 ; Gnan et Luedecke, 1982 ; Firstenderg et Tricarico, 1983 ; Fir stenberg-Eden, 1984 ; Suhren et Heeschen, 1985 ; Nieuwenhof et Hoolwer-', 1987). Mais les conditions expérimentales (milieu de culture et/ou température d'incubation, type de matériel) et les paramètres statistiques d'évaluation varient souvent d'une étude à l'autre. En l'absence de recommandations normalisées, il est alors difficile de comparer les résultats et de porter un jugement objectif sur la valeur de la technique. Cependant, certaines conditions expérimen- 
tales semblent mériter une comparaison : l'inoculation de l'échantillon à la surface d'un milieu gélosé (FIRSTEnBERG et Tricarico, 1983) et son incorporation dans la gélose (NieuWenHof et HoOlWerf, 1987) ; une température d'incubation de $18{ }^{\circ} \mathrm{C}$ (Firstenberg et Tricarico, 1983) et de $30^{\circ} \mathrm{C}$ (Suhren et Heeschen, 1985 et 1987). Le premier objectif de notre travail a donc été de déterminer expérimentalement, sur le Bactometer M 123, toutes les caractéristiques analytiques des mesures de conductance dans ces différentes conditions expérimentales. Ensuite, parmi les facteurs susceptibles de modifier ces caractéristiques, certains ont déjà été étudiés, comme la nature de la flore (FIrSTEnBERG et TriCARICO, 1983 ; Suhren et HeEschen, 1985 et 1987). Dans la seconde partie de nos travaux, nous nous sommes donc limités à l'étude de l'effet de deux autres facteurs sur la précision des mesures : le degré de dispersion des amas microbiens et l'origine des échantillons.

\section{Matériel et méthodes}

\section{A. Origine des échantillons}

Deux cent quatre-vingt-sept échantillons de lait cru ont été prélevés et conservés à $0^{\circ} \mathrm{C}$, au plus 24 heures après le prélèvement. Parmi ces échantillons, 134 provenaient du mélange non réfrigéré du lait d'une traite (laits de producteurs), 69 du mélange habituel du lait de quatre traites (laits de tank) et 44 de citernes de ramassage de deux entreprises laitières (laits de citerne).

Afin d'obtenir des laits contenant très peu de bactéries, 20 échantillons de lait ont été prélevés et partiellement écrémés après remontée de la matière grasse à $0^{\circ} \mathrm{C}$ pendant 24 heures (laits épurés). D'autre part, 40 échantillons de lait cru de très mauvaise qualité bactériologique ont été obtenus après une incubation de 12 heures à $18^{\circ} \mathrm{C}$ et un refroidissement à $0^{\circ} \mathrm{C}$ pour stopper la croissance (laits incubés).

\section{B. Mode d'agitation des échantillons}

Chaque échantillon a été soumis, avant analyse, à une agitation standard consistant en 25 mouvements aller-retour d'une amplitude de $25-30 \mathrm{~cm}$ effectués en 7 à 10 secondes (Anon., 1972).

Par ailleurs, pour étudier l'incidence du mode d'agitation des échantillons sur les caractéristiques analytiques des mesures d'impédance, 40 échantillons ont été répartis, après homogénéisation, en deux fractions de $25 \mathrm{ml}$ dans des flacons de $60 \mathrm{ml}$ : l'une d'elles a subi une agitation standard et l'autre fraction une agitation pendant 30 secondes à l'aide d'un homogénéisateur à turbine (Ultra-Turrax, type 18/10) tournant à 20000 tours/minute. Ce dernier mode d'agitation est actuellement celui qui assure la meilleure dispersion des amas microbiens (RICHARD, 1980 ; RiCHARD et al., 1981).

\section{Numérations microbiennes}

Les échantillons de lait cru ont été soumis au dénombrement des bactéries aérobies mésophiles sur milieu Plate Count Agar (Difco) après 3 jours d'incuba- 
tion à $30^{\circ} \mathrm{C}$. Les ensemencements ont été réalisés à la surface de la gélose à l'aide de l'appareil Spiral décrit par GiLchrist et al. (1973). Nous avons choisi cette méthode comme technique de référence car il a été largement montré qu'elle donne des résultats similaires à la technique classique de dénombrement (CAMPBell et GilChrist, 1973 ; GilChrist et al., 1973 ; DonNelly et al., 1976 ; Jarvis et al., 1977 ; PeELER et al., 1977).

Les analyses ont été effectuées en double pour chaque échantillon, afin d'obtenir une plus grande précision des résultats de dénombrement. Les résultats sont exprimés en unités formant colonies par millilitre de lait (UFC/ml).

\section{Mesures de conductance}

\section{Description de l'appareil Bactometer}

Le Bactometer Microbial Monitoring System M 123 (Bactomatic LTD, Henleyon-Thames) a déjà été largement décrit par FirstenBERG-EdEN et EDEN (1984). Toutefois, nous rappellerons ici ses principales caractéristiques. C'est un appareil composé de deux parties principales :

- une unité de mesure (Bactometer Processing Unit ou BPU) capable d'analyser simultanément 128 échantillons. Elle comprend deux incubateurs indépendants, réglés à des températures pouvant varier entre 10 et $55^{\circ} \mathrm{C}$. Les échantillons sont répartis dans les cuvettes de modules à usage unique (Bactomatic); chacune des 16 cuvettes d'un module contient deux électrodes de métal qui sont connectées au circuit électrique dans l'incubateur. Les mesures sont effectuées toutes les 6 minutes pour chaque échantillon ;

- un ordinateur pour le contrôle de l'unité de mesure, l'acquisition et l'analyse des données. En particulier, un algorithme permet de déterminer automatiquement, pour chaque échantillon, le temps de détection, c'est-à-dire le temps au bout duquel on peut mesurer une augmentation significative de la conductance dans la cuvette (fig. 1) ; ces temps de détection sont alors affichés à l'écran.

\section{Utilisation du Bactometer}

Le milieu MPCA préconisé par FIRSTENBERG et Tricarico (1983) est réparti stérilement dans les modules, à raison de $0,5 \mathrm{ml} /$ cuvette. Ce milieu a la composition suivante (en $\mathrm{g} / \mathrm{l}$ ) : extrait de levure $20 \mathrm{~g}$; tryptone $20 \mathrm{~g}$; glucose $4 \mathrm{~g}$; gélose $10 \mathrm{~g}$. Après solidification, l'échantillon de lait agité est déposé à la surface de la gélose à raison de $0,1 \mathrm{ml} /$ cuvette et les modules sont placés immédiatement dans les incubateurs réglés à $18{ }^{\circ} \mathrm{C}$ ou à $30^{\circ} \mathrm{C}$.

Nous avons également testé sur 134 échantillons la technique d'ensemencement des modules préconisée par NieuwenHoF et Hoolwerf (1987). Celle-ci consiste à mélanger $2 \mathrm{ml}$ de l'échantillon à analyser avec $10 \mathrm{ml}$ de milieu MPCA fondu et refroidi à $45^{\circ} \mathrm{C}$. Le mélange est ensuite réparti dans la cuvette de mesure à raison de $0,6 \mathrm{ml} /$ cuvette. Après solidification du milieu, les modules sont placés dans l'incubateur.

Les temps de détection d'un changement significatif de conductance ont été déterminés automatiquement par l'appareil. Cependant, après examen visuel des 
courbes, nous avons éliminé les résultats pour lesquels l'appareil a réalisé une mauvaise détection en raison d'une irrégularité dans la phase initiale de la courbe (fig. 1).

\section{E. Calculs statistiques}

La répétabilité et la justesse des mesures de conductance ont été calculées conformément aux recommandations de la norme FIL 128 (Ánon., 1985) ; avant les calculs statistiques, les nombres d'UFC/ml ont subi une transformation logarithmique pour normaliser la distribution.

\section{- Justesse}

La justesse représente l'étroitesse de l'accord entre la valeur vraie de la grandeur à mesurer et le résultat moyen qui serait obtenu en appliquant la technique expérimentale un grand nombre de fois (Anon., 1985). L'évaluation de la justesse d'une technique revient à comparer la valeur vraie de la grandeur à mesurer avec la valeur moyenne de plusieurs déterminations obtenues avec la technique testée, de façon à réduire les erreurs de fidélité. Par convention, la valeur vraie est donnée par le nombre d'UFC/ml obtenu par les techniques classiques de dénombrement (dans notre cas, moyenne des deux déterminations effectuées avec l'appareil Spiral).

Les résultats antérieurs ont déjà clairement établi que, pour les niveaux habituels de contamination microbienne du lait cru (moins de $10^{6}$ à $10^{7} \mathrm{UFC} / \mathrm{ml}$ ), il existe une relation linéaire entre le temps de détection et le logarithme du nombre d'UFC $/ \mathrm{ml}$. Conformément à la norme FIL, l'équation de calibrage $(\mathrm{y}=\mathrm{b} \cdot \mathrm{x}+\mathrm{a})$ a été établie selon la méthode des moindres carrés à partir des moyennes des deux déterminations faites sur chaque échantillon par les deux techniques ; les logarithmes décimaux des nombres d'UFC/ml sont considérés comme variable dépendante et les temps de détection comme variable indépendante, pour donner une estimation de l'écart-type résiduel dans l'unité de la méthode de référence. Nous avons appliqué la norme FIL établie pour les méthodes chimiques bien que certaines hypothèses ne soient plus vérifiées pour les techniques microbiologiques (en particulier, une erreur négligeable dans la mesure des $\mathrm{x}$ et des $\mathrm{y}$ ).

La précision d'estimation de la technique expérimentale est donnée par les limites de l'intervalle de confiance à l'intérieur duquel se situe, avec une probabilité de $95 \%$, la vraie valeur donnée par la méthode de référence. Si y est le nombre d'UFC/ml calculé à partir de la droite de calibrage, ces limites sont, en valeur logarithmique, $\log y \pm 1,96 . s_{y, x}$ où $s_{y, x}$ est l'écart-type résiduel des écarts à la droite de régression

$$
s_{y, x}=\sqrt{\frac{1}{n-2} \sum_{i=1}^{n}\left(y_{i}-\hat{y}_{i}\right)^{2}}
$$

$y_{i}$ et $\hat{y}_{i}$ étant respectivement les valeurs observées et calculées de la technique de référence pour chaque échantillon i. En données non transformées, ces limites sont $\mathrm{y} / 10^{1,96.5} \mathrm{y}, \mathrm{x}$ pour la limite inférieure et $\mathrm{y} \cdot 10^{1,96.5} \mathrm{y}, \mathrm{x}$ pour la limite supérieure. 


\section{- Répétabilité}

La variance de répétabilité des temps de détection $\left(\mathrm{s}_{\mathrm{r}}{ }^{2}\right)$ a été établie à partir des mesures de conductance effectuées en double sur chaque échantillon, selon la formule suivante :

$$
\mathrm{s}_{\mathrm{r}}^{2}=\frac{1}{2 \mathrm{n}} \sum_{\mathrm{i}=1}^{\mathrm{n}} \mathrm{w}_{\mathrm{i}}^{2}
$$

où $w_{i}$ est la valeur absolue de la différence entre doubles pour l'échantillon $\mathrm{i}$ (Anon., 1985). Dans le cas des temps de détection, l'écart-type de répétabilité est alors exprimé en heures ; mais pour pouvoir comparer valablement cet écart-type avec celui des autres méthodes, nous l'avons également exprimé en $\log _{10} \mathrm{UFC} / \mathrm{ml}$ grâce à la formule :

$$
\mathrm{s}_{\mathrm{r}} *\left(\mathrm{en} \log _{10} \mathrm{UFC}\right)=|\mathrm{b}| . \mathrm{s}_{\mathrm{r}}
$$

où $\mathrm{b}$ est la pente de la droite de calibrage.

Le coefficient de variation de répétabilité $\mathrm{CV}_{\mathrm{r}}{ }^{*}$ en données non transformées peut alors être estimé à partir de la formule suivante :

$$
\mathrm{CV}_{\mathrm{r}} *(\mathrm{en} \mathrm{UFC})=\left(10 \mathrm{~s}_{\mathrm{r}} *-1\right) \cdot 100
$$

La figure 2 présente l'intérêt d'une telle conversion de l'écart-type de répétabilité en UFC/ml ; en effet, deux techniques qui donnent le même écart-type de répétabilité exprimé dans l'unité d'origine, ont en fait des répétabilités exprimées en $\mathrm{UFC} / \mathrm{ml}$ différentes si les pentes des équations de calibrage sont différentes.

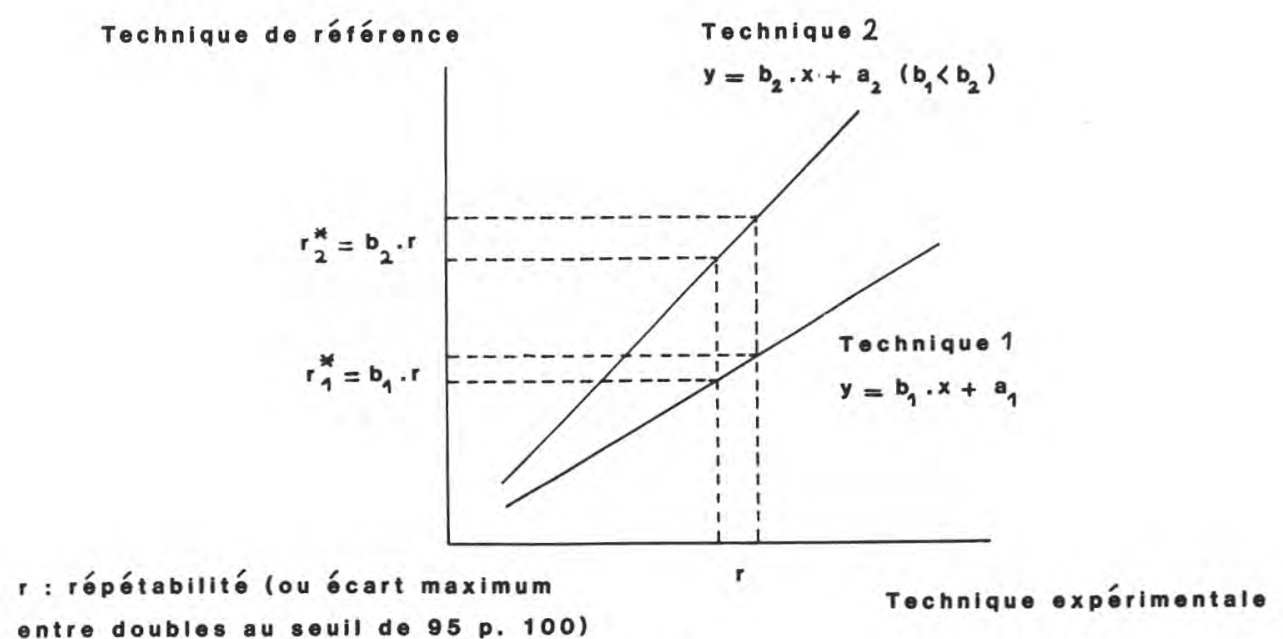

Fig. 2

Conversion de la répétabilité d'une technique expérimentale dans l'unité de la technique de référence.

Expression of the test method repeatability in the reference method unit. 
Enfin, on peut démontrer mathématiquement (Firstenberg-Eden et Eden, 1984) que le coefficient de régression de la droite de calibrage permet d'estimer le temps de génération moyen $\left(\mathrm{t}_{\mathrm{g}}\right)$ de la flore microbienne selon la formule :

$$
t_{g}=\frac{\log 2}{b}
$$

\section{Résultats}

A. Comparaison des températures de 30 et $18{ }^{\circ} \mathrm{C}$

La figure 3 montre les relations obtenues à 30 et $18{ }^{\circ} \mathrm{C}$ entre le temps de détection et le logarithme décimal du nombre de micro-organismes/ml de lait ; ces rela-

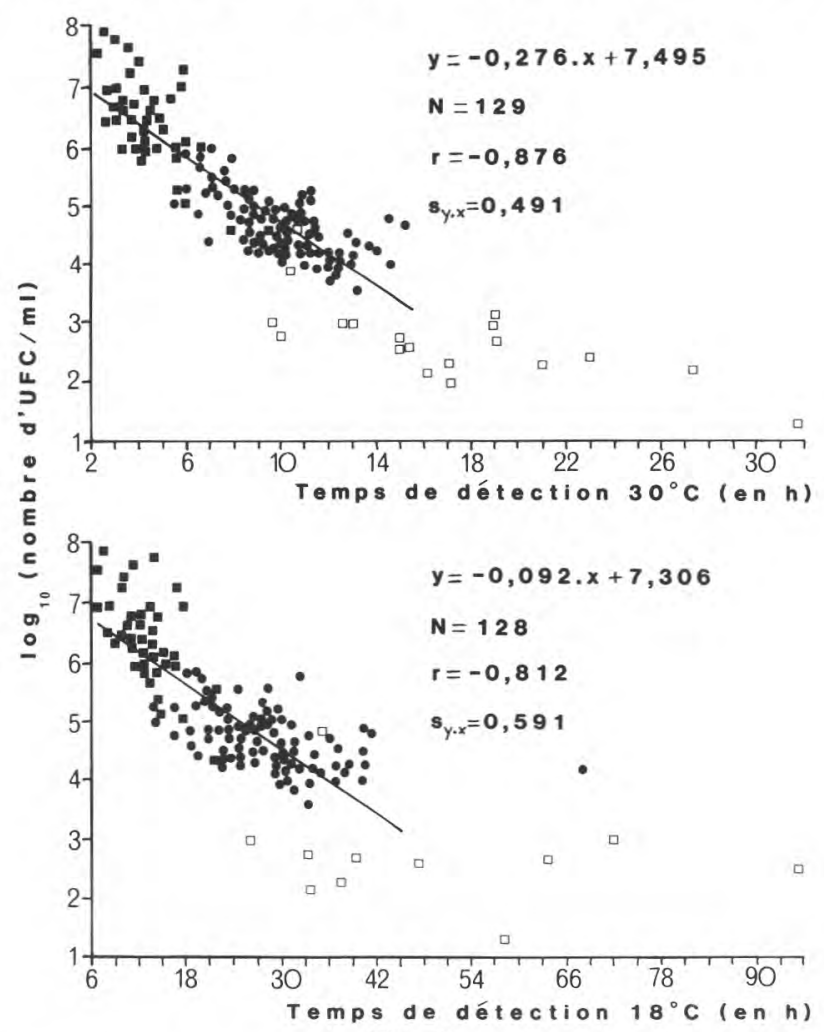

Fig. 3

Relation entre les temps de détection à $30^{\circ} \mathrm{C}$ et $18^{\circ} \mathrm{C}$ et les résultats obtenus par la méthode de référence.

(- laits incubés ; $(\square)$ laits épurés; $(\bullet)$ laits de producteurs.

Les droites de calibrage ont été calculées après élimination des échantillons de lait épurés à $30^{\circ} \mathrm{C}$ et à $18{ }^{\circ} \mathrm{C}$, d'un échantillon qui s'écarte de plus de 3 écarts-type résiduels de ces droites.

Relationship between the detection times at $30^{\circ} \mathrm{C}$ and $18{ }^{\circ} \mathrm{C}$ and the results given by the reference method $(C F U / \mathrm{ml})$.

(畐) milk samples incubated during 12 hours à $18{ }^{\circ} \mathrm{C}$; $(\square)$ milk samples of good bacteriological quality obtained by creaming; (๑) farm milks (mixture of one milking). 
tions ont été établies à partir d'une population identique d'échantillons comprenant 20 laits épurés, 40 laits incubés et 90 laits de producteurs. La courbe d'ajustement de forme parabolique ne peut être assimilée à une droite qu'audessus de $10^{3}$ à $10^{4} \mathrm{UFC} / \mathrm{ml}$; on constate également une déviation par rapport au modèle linéaire au-dessus de $10^{7} \mathrm{UFC} / \mathrm{ml}$, conformément au modèle théorique (Firstenterg-Eden et Eden, 1984). L'équation de calibrage a été calculée après élimination des laits épurés par crèmage. La précision d'estimation des mesures de conductance semble plus satisfaisante à $30^{\circ} \mathrm{C}$ qu'à $18^{\circ} \mathrm{C}$ : celle-ci passe en effet d'environ $\pm 1,0$ à $\pm 1,2 \log \mathrm{UFC} / \mathrm{ml}$. Cette différence est d'ailleurs significative au seuil de $5 \%$ (test $\mathrm{F}$ d'égalité des variances à n-2 ddl; Dagnelie, 1970).

Compte tenu de l'inégalité des variances résiduelles, nous n'avons pas comparé statistiquement les pentes des droites de régression. Cependant, on peut observer que les temps de génération de la flore semblent nettement plus courts à $30^{\circ} \mathrm{C}$ qu'à $18^{\circ} \mathrm{C}$.

Pour les échantillons de lait contenant plus de $10^{4} \mathrm{UFC} / \mathrm{ml}$, le coefficient de variation de répétabilité (en heures) des temps de détection est de $5,3 \%$ à $18{ }^{\circ} \mathrm{C}$ et de $6,3 \%$ à $30{ }^{\circ} \mathrm{C}$. Après conversion en $\mathrm{UFC} / \mathrm{ml}$ par l'intermédiaire des pentes des droites de calibrage, les écarts-type de répétabilité $\left(\mathrm{s}_{\mathrm{r}}{ }^{*}\right)$ sont, en unités logarithmiques, de 0,110 à $18{ }^{\circ} \mathrm{C}$ et de 0,143 à $30^{\circ} \mathrm{C}$, soit respectivement des coefficients de variation de 28,8 et $39 \%$ en données non transformées.

\section{B. Comparaison de deux modes d'inoculation des échantillons dans les cuvettes}

Sur le tableau 1, sont représentées les relations obtenues entre le temps de détection à $30^{\circ} \mathrm{C}$ et le logarithme du nombre d'UFC/ml, pour deux modes d'inoculation des échantillons de lait dans les cuvettes de mesure : à la surface ou dans la masse du milieu gélosé. Ces relations ont été établies à partir d'une même popu-

\section{TABLEAU 1}

Influence du mode d'inoculation des échantillons de lait dans les cuvettes sur la relation entre les temps de détection à $30{ }^{\circ} \mathrm{C}$ et les résultats de la méthode de référence.

Influence of the sample inoculation method in the modules on the relationship between the detection times at $30{ }^{\circ} \mathrm{C}$ and the reference plate counts (CFU/ml)

\begin{tabular}{|c|c|c|c|c|c|}
\hline $\begin{array}{c}\text { Mode } \\
\text { d'inoculation }\end{array}$ & $\mathrm{N}^{(\mathrm{a})}$ & $\begin{array}{l}\text { Equation } \\
\text { de } \text { régression }^{(b)}\end{array}$ & $\mathrm{r}^{(\mathrm{c})}$ & $s_{y, x}{ }^{(d)}$ & $\begin{array}{c}\text { Temps } \\
\text { moyen } \\
\text { de } \\
\text { détection }\end{array}$ \\
\hline Surface & 134 & $y=-0,200 x+6,777$ & $-0,676$ & 0,465 & 9,6 \\
\hline Masse & 134 & $y=-0,177 x+6,345$ & $-0,626$ & 0,492 & 8,4 \\
\hline
\end{tabular}
(a) N : Nombre d'échantillons.
(b) $y: \log _{10}$ du nombre d'UFC/ml; $\mathrm{x}$ : temps de détection en heures.
(c) $\mathrm{r}$; coefficient de corrélation.
(d) $s_{y, x}$ : écart-type résiduel. 
lation de 134 échantillons de lait de producteurs. La précision d'estimation semble légèrement meilleure dans le cas de l'ensemencement en surface mais la différence n'est pas significative (test $\mathrm{F}$ de comparaison des variances à $\mathrm{n}-2$ ddl ; DAGNELIE, 1970). Cette précision est par ailleurs très proche de celle que nous avons établie dans l'essai précédent sur une autre population d'échantillons, soit $\pm 0,90$ à $1,0 \mathrm{log} \mathrm{UFC} / \mathrm{ml}$. La pente de la droite de calibrage est, en valeur absolue, significativement plus faible pour l'inoculation dans la masse de la gélose, au seuil de $5 \%$ (test $\mathrm{t}$ d'égalité de deux coefficients de régression; Dagnelie, 1970). On peut calculer que le temps de génération moyen de la flore présente dans ces échantillons passe de $1,51 \mathrm{~h}$ dans le cas d'une inoculation en surface à $1,70 \mathrm{~h}$ pour l'ensemencement dans la masse. Par ailleurs, les temps de détection sont, en moyenne, significativement plus courts dans la masse qu'en surface au seuil de $5 \%$ (test t par paires; Dagnelie, 1970).

On observe sur le tableau 2 qu'en moyenne, la répétabilité des temps de détection est plus satisfaisante dans le cas de l'ensemencement dans la masse. Ainsi, quel que soit le niveau de contamination du lait, l'écart-type de répétabilité des temps de détection (en heures) est toujours inférieur avec ce mode d'ensemencement. D'autre part, on peut noter que cet écart type augmente lorsque le niveau de contamination du lait baisse. Après conversion en UFC, cette augmentation est particulièrement nette avec l'inoculation en surface : ainsi, au-dessous de $5.10^{4}$ $\mathrm{UFC} / \mathrm{ml}$., le $\mathrm{CV}_{\mathrm{r}}$ en UFC/ml dépasse largement $30 \%$.

TABLEAU 2

Comparaison de la répétabilité des temps de détection obtenus à $30^{\circ} \mathrm{C}$ avec deux modes d'inoculation des échantillons de lait dans les cuvettes.

Comparison of the repeatability of the detection times obtained at $30{ }^{\circ} \mathrm{C}$ with two inoculation methods of the milk samples in the wells.

\begin{tabular}{|c|c|c|c|c|c|c|c|}
\hline \multirow{2}{*}{$\begin{array}{c}\text { Mode } \\
\text { d'inoculation }\end{array}$} & \multirow{2}{*}{$\begin{array}{c}\text { Classe } \\
\text { (en } \mathrm{UFC} / \mathrm{ml} \text { ) }\end{array}$} & \multirow{2}{*}{$\begin{array}{l}\text { Equivalence } \\
\text { (en heures) }\end{array}$} & \multirow{2}{*}{$N^{(a)}$} & \multicolumn{2}{|c|}{$\begin{array}{l}\text { Répétabilité } \\
\text { en heures }\end{array}$} & \multicolumn{2}{|c|}{$\begin{array}{l}\text { Répétabilité } \\
\text { en UFC } / \mathrm{ml}\end{array}$} \\
\hline & & & & $s_{r}$ & $\mathrm{CV}_{\mathrm{r}}$ & $\mathrm{S}_{\mathrm{r}}^{*}$ & $\mathrm{CV}_{\mathrm{r}}{ }^{*}$ \\
\hline \multirow[t]{4}{*}{ Surface } & $>10^{5}$ & $<8,8$ & 33 & 0,36 & 5,0 & 0,072 & 17,9 \\
\hline & $5.10^{4}-10^{5}$ & $8,8-10,4$ & 30 & 0,41 & 4,2 & 0,082 & 20,7 \\
\hline & $<5.10^{4}$ & $>10,4$ & 27 & 0,66 & 5,5 & 0,132 & 35,6 \\
\hline & Total & & 90 & 0,48 & 5,1 & 0,097 & 25,0 \\
\hline \multirow[t]{4}{*}{ Masse } & $>10^{5}$ & $<7,6$ & 33 & 0,25 & 4,2 & 0,044 & 10,8 \\
\hline & $5.10^{4}-10^{5}$ & $7,6-9,3$ & 30 & 0,26 & 3,1 & 0,047 & 11,3 \\
\hline & $<5.10^{4}$ & $>9,3$ & 27 & 0,38 & 3,4 & 0,066 & 16,5 \\
\hline & Total & & 90 & 0,30 & 3,6 & 0,053 & 12,9 \\
\hline
\end{tabular}

(a) $\mathrm{N}$ : nombre d'échantillons. 


\section{Influence de l'origine des échantillons}

L'influence de l'origine des échantillons de lait sur les caractéristiques analytiques de la technique expérimentale a été établie à partir de l'analyse de 243 échantillons de lait cru : 90 laits de producteurs, 69 laits de tank et 44 laits de citerne. Les conditions expérimentales retenues pour les mesures de conductance ont alors été les suivantes : l'ensemencement du lait dans les cuvettes à la surface du milieu gélosé et une température d'incubation de $30^{\circ} \mathrm{C}$.

Le tableau 3 présente la répétabilité des temps de détection en fonction du niveau de contamination microbienne du lait. Conformément à ce qui a été observé précédemment, on note un coefficient de variation de répétabilité (en heures) constant pour des niveaux supérieurs à $10^{5} \mathrm{UFC} / \mathrm{ml}$; par contre, il augmente de façon très importante au-dessous de ce seuil. Les mêmes conclusions peuvent être effectuées si on exprime les valeurs de répétabilité en UFC/ml. D'autre part, on note que les coefficients de variation de répétabilité sont nettement plus élevés lorsqu'ils sont exprimés dans l'unité de la technique de référence.

\section{TABLEAU 3}

Répétabilité des temps de détection obtenue à $30^{\circ} \mathrm{C}$ avec une inoculation en surface, en fonction du niveau de contamination des échantillons de lait

Repeatability of the detection times obtained at $30{ }^{\circ} \mathrm{C}$ with an inoculation of the sample at the medium surface

\begin{tabular}{l|c|c|c|c|c|c}
\hline \multirow{2}{*}{$\begin{array}{c}\text { Classe } \\
\text { en } \\
\text { heures }\end{array}$} & $\begin{array}{c}\text { Equivalence en } \\
\text { nombre d'UFC/ml } \\
\left(\mathrm{x} 10^{3}\right)(\mathrm{b})\end{array}$ & \multirow{2}{*}{$\mathrm{N}^{(\mathrm{a})}$} & \multicolumn{2}{c|}{$\begin{array}{c}\text { Répétabilité } \\
\text { en heures }\end{array}$} & \multicolumn{2}{c}{$\begin{array}{c}\text { Répétabilité } \\
\text { en UFC/ml }\end{array}$} \\
\cline { 4 - 8 } & & & $\mathrm{s}_{\mathrm{r}}$ & $\mathrm{CV}_{\mathrm{r}}$ & $\mathrm{s}_{\mathrm{r}}{ }^{*}$ & $\mathrm{CV}_{\mathrm{r}}{ }^{*}$ \\
\hline$<6$ & $>290$ & 36 & 0,234 & 5,7 & 0,045 & 10,9 \\
$6-8$ & $120-290$ & 51 & 0,366 & 5,1 & 0,070 & 17,5 \\
$8-10$ & $50-120$ & 72 & 0,615 & 6,9 & 0,117 & 30,9 \\
$10-12$ & $20-50$ & 49 & 1,259 & 11,6 & 0,239 & 73,4 \\
$>12$ & $<20$ & 35 & 2,155 & 14,4 & 0,410 & 157,0 \\
\hline Total & & 243 & 1,072 & 11,8 & 0,204 & 60,0 \\
\hline
\end{tabular}

(a) $\mathrm{N}$ : nombre d'échantillons.

(b) Nombre d'UFC/ml calculé à partir de l'équation de calibrage.

La relation entre les temps de détection et les nombres d'UFC/ml (en $\left.\log _{10}\right)$ est représentée sur la figure 4. L'équation de la droite de calibrage calculée sur les 243 échantillons contenant entre $4.10^{3}$ et $4.10^{6} \mathrm{UFC} / \mathrm{ml}$ est la suivante :

$$
\log _{10} y=-0,191 \cdot x+6,605
$$

avec un écart type résiduel $\left(\mathrm{s}_{\mathrm{y}, \mathrm{x}}\right)$ de 0,441 et un coefficient de corrélation linéaire (r) de $-0,657$. La précision d'estimation de la technique est donc de $\pm 1,96.0,441$, 


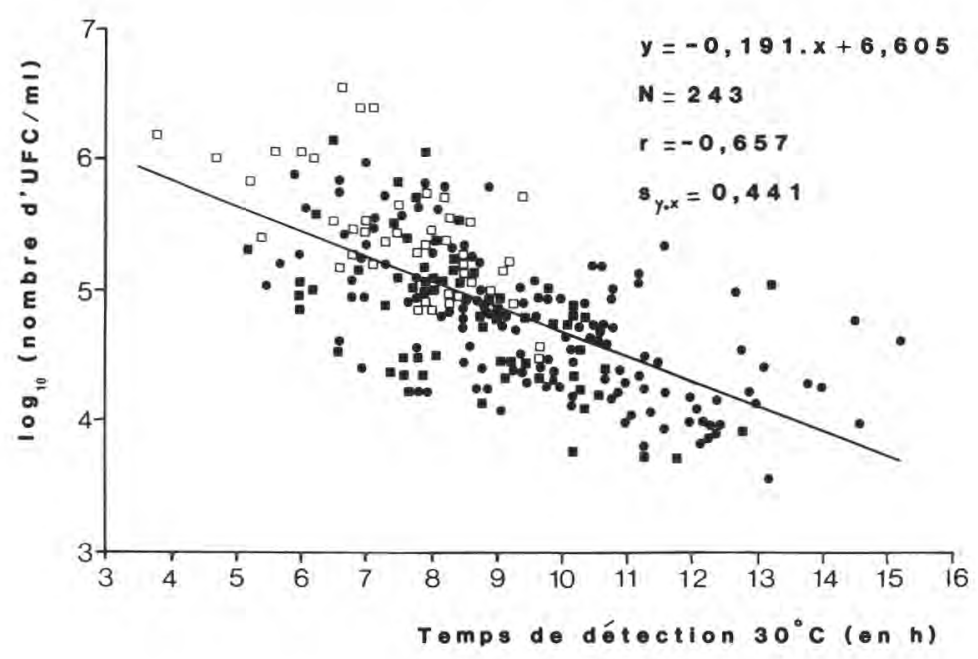

Fig. 4

Relation entre les temps de détection à $30{ }^{\circ} \mathrm{C}$ avec une inoculation en surface et les résultats de la méthode de référence.

( ) laits de producteurs; ( $\mathbf{E})$ laits de tank; ( $\square$ ) laits de citerne.

Relationship between the reference standard plate counts $(C F U / m l)$ and the detection times obtained at $30{ }^{\circ} \mathrm{C}$ with an inoculation at the medium surface.

(-) farm milks (mixture of one milking); (回) farm milks (mixture of four milkings); ( $\square$ ) bulked tanker milks.

soit $\pm 0,864 \log _{10} \mathrm{UFC} / \mathrm{ml}$. Si y est le nombre d'UFC/ml calculé à partir de la droite de calibrage, les limites de l'intervalle de confiance de y (à $\mathrm{P}=0,95)$ sont en données non transformées :

$$
\begin{aligned}
& \text { y/ } 10^{0,864} \text { soit } y / 7,3 \text { pour la limite inférieure } \\
& \text { y. } 10^{0,864} \text { soit y. } 7,3 \text { pour la limite supérieure. }
\end{aligned}
$$

Sur le tableau 4, nous avons noté les caractéristiques de justesse des mesures de conductance établies pour les 3 classes d'échantillons : laits de producteurs, de tanks ou de citernes de ramassage. D’une façon générale, celles-ci varient peu en fonction de l'origine des échantillons. La précision d'estimation de la technique semble légèrement améliorée dans le cas des laits de citerne : celle-ci est alors de $\pm 0,72 \log \mathrm{UFC} / \mathrm{ml}$. Si y est le nombre d'UFC/ml calculé à partir de la droite de calibrage, les limites de l'intervalle de confiance de y sont y/5,3 pour la limite inférieure et y.5,3 pour la limite supérieure. Néanmoins, les trois variances résiduelles sont homogènes (test $\mathrm{F}$ de comparaison des variances à $\mathrm{n}-2 \mathrm{ddl}$; DAGNELIE, 1970). Si les coefficients de régression établis pour les laits de producteurs et les laits de tank ne sont pas significativement différents au seuil de $5 \%$, celui obtenu avec les laits de citerne est, en valeur absolue, significativement supérieur au seuil de $5 \%$ (test d'égalité des coefficients de régression; DAGNELIE, 1970). On peut calculer que le temps de génération moyen de la flore peut être estimé à $1,22 \mathrm{~h}$ pour les laits de citerne et à $1,84 \mathrm{~h}$ pour les laits de troupeaux. 


\section{TABLEAU 4}

Influence de l'origine des échantillons sur la relation entre les temps de détection à $30{ }^{\circ} \mathrm{C}$ avec une inoculation en surface, et les résultats de la méthode de référence

Influence of the origin of the milk samples on the relationship between the detection times obtained at $30^{\circ} \mathrm{C}$ with an inoculation at the medium surface, and the reference plate counts.

\begin{tabular}{l|r|c|c|c|c|c}
\hline \multirow{2}{*}{ Origine } & $\mathrm{N}^{(\mathrm{a})}$ & Équation de régression & & & & \multicolumn{2}{c|}{$\mathrm{r}^{(\mathrm{c})}$} & $\mathrm{s}_{\mathrm{y}, \mathrm{x}}{ }^{(\mathrm{d})}$ & \multicolumn{2}{c}{ Moyennes $^{(\mathrm{b})}$} \\
\cline { 5 - 7 } & & & & & $\mathrm{x}$ & $\mathrm{y}$ \\
\hline Producteurs & 130 & $\mathrm{y}=-0,168 \mathrm{x}+6,353$ & $-0,646$ & 0,412 & 9,71 & 4,72 \\
Tank & 69 & $\mathrm{y}=-0,160 \mathrm{x}+6,228$ & $-0,514$ & 0,468 & 8,61 & 4,85 \\
Citernes & 44 & $\mathrm{y}=-0,247 \mathrm{x}+7,297$ & $-0,633$ & 0,367 & 7,65 & 5,41 \\
\hline
\end{tabular}

(a) N : Nombre d'échantillons

(b) $y: \log _{10}$ du nombre d'UFC/ml ; $\mathrm{x}$ : temps de détection en heures

(c) $\mathrm{r}$ : coefficient de corrélation

(d) $\mathrm{s}_{\mathrm{y}, \mathrm{x}}$ : écart-type résiduel

\section{TABLEAU 5}

Influence du mode d'agitation des échantillons de lait sur la relation entre les temps de détection à $30{ }^{\circ} \mathrm{C}$ avec une inoculation en surface et les résultats de la méthode de référence

Influence of the sample shaking method on the relationship between the reference plate counts and the detection times at $30^{\circ} \mathrm{C}$ with an inoculation at the medium surface

\begin{tabular}{l|c|c|c|c|c|c}
\hline \multirow{2}{*}{$\begin{array}{c}\text { Mode } \\
\text { d'agitation }\end{array}$} & $\mathrm{N}^{(\mathrm{a})}$ & Équation de régression & & $\mathrm{r}^{(\mathrm{c})}$ & $\mathrm{s}_{\mathrm{y}, \mathrm{x}}{ }^{(\mathrm{d})}$ & \multicolumn{2}{|c}{ Moyennes $^{(\mathrm{b})}$} \\
\cline { 5 - 7 } & & & & $\mathrm{x}$ & $\mathrm{y}$ \\
\hline $\begin{array}{l}\text { Standard } \\
\text { Turbine }\end{array}$ & 40 & $\mathrm{y}=-0,162 \mathrm{x}+6,294$ & $-0,525$ & 0,496 & 9,13 & 4,82 \\
& 40 & $\mathrm{y}=-0,227 \mathrm{x}+7,213$ & $-0,515$ & 0,549 & 8,86 & 5,21 \\
\hline
\end{tabular}

(a) $\mathrm{N}$ : Nombre d'échantillons

(b) $\mathrm{y}: \log _{10} \mathrm{du}$ nombre $\mathrm{d}^{\prime} \mathrm{UFC} / \mathrm{ml}$; $\mathrm{x}:$ temps de détection en heures

(c) $\mathrm{r}$ : coefficient de corrélation

(d) $\mathrm{s}_{\mathrm{y}, \mathrm{x}}$ : écart-type résiduel

D. Incidence du mode d'agitation des échantillons sur la relation entre les mesures de conductance et la technique de référence

Le tableau 5 présente les caractéristiques analytiques des mesures de conductance établies à partir de l'analyse de 40 échantillons de lait cru, après agitation standard d'une part et après agitation violente à l'aide d'une turbine d'autre part. On observe tout d'abord que les variances résiduelles ne diffèrent pas significative- 
ment au seuil de $5 \%$ (test $\mathrm{F}$ de comparaison des variances : DAgNelie, 1970); il en est de même des coefficients de régression. Par contre, les temps de détection sont significativement plus courts avec l'agitation violente (test $t$ par paires ; DaGNELIE, 1970) ; la différence est cependant très faible (moins de $0,3 \mathrm{~h}$ en moyenne). De même, les nombres d'UFC/ml sont significativement plus élevés avec l'agitation violente au seuil de $5 \%$ : en moyenne de 0,4 unité de log.

\section{Discussion et conclusions}

Les résultats de notre étude permettent de définir les conditions expérimentales qui semblent assurer aux mesures d'impédance, les caractéristiques analytiques les plus performantes, soit une inoculation de l'échantillon de lait dans la masse du milieu gélosé et une température d'incubation de $30^{\circ} \mathrm{C}$ (tabl. 6). Tout d'abord, la température de $30^{\circ} \mathrm{C}$ présente l'avantage de temps de détection plus courts (en moyenne 8 à 9 heures contre près de 24 heures pour $18^{\circ} \mathrm{C}$ ). Ensuite, si la température de $18{ }^{\circ} \mathrm{C}$ donne une meilleure répétabilité des temps de détection, la précision d'estimation des mesures de conductance est plus satisfaisante à $30^{\circ} \mathrm{C}$. Ces observations sont en contradiction avec celles de FirSTENBERG-EDEN et Tricarico (1983) : en effet, d'après ces auteurs, une température de $18^{\circ} \mathrm{C}$ est préférable car les temps de génération mesurés sur 14 souches bactériennes présentent une variabilité minimale à $18{ }^{\circ} \mathrm{C}$, à l'intérieur d'une plage de 15 à $30^{\circ} \mathrm{C}$. A l'inverse, d'après SuHREn et HeEschen $(1985,1987)$, cette variabilité pour 13 souches isolées du lait ou de produits laitiers est plus faible à $30^{\circ} \mathrm{C}$ qu'à $20^{\circ} \mathrm{C}$. Cette contradiction entre auteurs provient certainement du choix des souches, les premiers ayant plutôt testé des souches psychrotrophes et les seconds à la fois des souches psychrotrophes et mésophiles. Compte tenu de la difficulté de disposer de souches représentatives de la flore bactérienne du lait cru (ThомAs et al., 1962 ; TWOMEY et CRAwley, 1968), il semble donc plus simple de réaliser le choix de la température d'incubation après analyse d'une population représentative des échantillons de lait habituellement analysés.

\section{TABLEAU 6}

Tableau récapitulatif des principales caractéristiques analytiques des mesures de conductance.

Summary of the main analytical attributes of the conductance measures.

\begin{tabular}{l|l|c|c|c|c|c}
\hline Température & $\begin{array}{c}\text { Mode } \\
\text { d'inoculation }\end{array}$ & $\mathrm{N}$ & $\mathrm{s}_{\mathrm{r}}{ }^{*}$ & $\mathrm{CV}_{\mathrm{r}}{ }^{*}$ & $\mathrm{~s}_{\mathrm{y}, \mathrm{x}}$ & $\begin{array}{c}\text { Temps } \\
\text { moyen } \\
\text { de } \\
\text { détection }\end{array}$ \\
\hline $30^{\circ} \mathrm{C}$ & Masse & 134 & 0,053 & 12,9 & 0,492 & 8,4 \\
& Surface & 129 & 0,143 & 39,0 & 0,491 & 8,3 \\
& & 134 & 0,097 & 25,0 & 0,465 & 9,6 \\
& Surface & 128 & 0,110 & 28,8 & 0,591 & 23,0 \\
\hline $18{ }^{\circ} \mathrm{C}$ & & & & & &
\end{tabular}


D'autre part, conformément aux conclusions de NIEUWENHOF et HoolwerF (1987), la répétabilité des temps de détection est nettement améliorée dans le cas de l'inoculation dans la masse par rapport à une inoculation en surface ; ce phénomène est particulièrement net pour les faibles niveaux de contamination microbienne du lait. Quelques hypothèses peuvent être évoquées comme par exemple, le prélèvement d'une plus grande quantité de lait $(1 \mathrm{ml}$ contre $0,1 \mathrm{ml})$ ou une meilleure homogénéité des variations de composition dans le milieu de culture. Malgré des temps de génération moyens de la flore plus longs, les temps de détection sont significativement plus courts lorsque l'inoculation est effectuée dans la masse de la gélose ; la différence relativement importante (en moyenne plus d'une heure) est probablement liée à une meilleure diffusion des métabolites dans le milieu et donc à un délai de réponse plus court. Enfin, la précision d'estimation des mesures de conductance semble identique avec les deux modes d'ensemencement. A l'inverse, NIEUWENHOF et HoOlWERF (1987) avaient obtenu une meilleure précision d'estimation avec une inoculation dans la masse du milieu gélosé. Ces conclusions divergentes des nôtres sont liées au fait que la comparaison réalisée par ces auteurs a été faite sur deux populations d'échantillons différentes; or, on a pu mettre en évidence dans notre étude (tabl. 6) que l'écart-type résiduel de la régression peut varier dans des proportions similaires (entre 0,40 et $0,50 \log \mathrm{UFC} / \mathrm{ml}$ ) entre essais effectués dans les mêmes conditions expérimentales sur deux populations différentes d'échantillons de laits de producteurs.

Un mode d'agitation violent des échantillons semble provoquer une réduction des temps de détection. La différence est cependant très faible ; cela tend à montrer que les résultats obtenus avec ce type de technique sont reliés au nombre réel de micro-organismes et non pas au nombre d'amas microbiens. Nous avons expliqué cette petite différence entre les deux modes d'agitation par la libération de micro-organismes agglutinés par exemple, avec les globules gras (BotTAZZI et al., 1972). Comme NiEUWENHOF et HoOLWERF (1987), nous n'avons pas observé d'amélioration de la précision d'estimation des mesures avec l'agitation violente des échantillons à l'aide d'une turbine ; cette conclusion est contraire à ce qu'on pouvait attendre, puisque ce mode d'agitation permet une désagrégation maximale des amas microbiens et donc une meilleure estimation du nombre de microorganismes présents (RICHARD, 1980). Cela vient probablement du fait que le changement de conductance du milieu est autant lié à l'activité des microorganismes dans le milieu de culture qu'à leur niveau réel initial dans le lait. Ainsi, il a été montré (Hadley et SenyK, 1975 ; Suhren et Heeschen, 1985 et 1987 ; FirSTENBERG-Eden et Eden, 1984) que le nombre de bactéries atteint dans la cuvette lors de ce changement varie dans des proportions assez larges (entre $10^{6}$ et $10^{8} / \mathrm{ml}$ ) ; en revanche, ce changement semble lié à une nette augmentation de la concentration en ammoniac dans le milieu, et donc à l'activité protéolytique microbienne dans ce milieu (SUHREN et HeEschen, 1985 et 1987). Il serait intéressant de connaître la relation des mesures d'impédance avec l'activité protéolytique antérieure des micro-organismes dans le lait et le degré de protéolyse des laits analysés ; ce sera l'objet d'une prochaine étude.

D'une façon générale, à $30^{\circ} \mathrm{C}$, les valeurs de répétabilité obtenues dans notre étude sont assez proches de celles qu'on peut trouver dans la littérature : l'écarttype de répétabilité peut atteindre $0,20 \mathrm{log} \mathrm{UFC} / \mathrm{ml}$ pour l'inoculation en surface ; à l'inverse, pour l'inoculation dans la masse et à tous les niveaux de contamination microbienne du lait, il est inférieur à 0,07 , la norme généralement admise pour les méthodes classiques de dénombrement (Anon,, 1972). O'ConNOR (1979) 
puis Nieuwenhof et Hoolwerf (1987) ont obtenu des répétabilités équivalentes dans les mêmes conditions expérimentales. Par contre, d'après FirstenBERG (1984), l'inoculation en surface permet d'obtenir une répétabilité aussi bonne, voire meilleure, que les techniques classiques. Mais cet auteur a analysé des échantillons d'une contamination supérieure à $9.10^{4} \mathrm{UFC} / \mathrm{ml}$; or, nous avons montré que c'est au-dessous de ce seuil que la répétabilité de cette technique se dégrade de façon importante.

Par ailleurs, on peut dire que la précision d'estimation des mesures de conductance à $30^{\circ} \mathrm{C}$ est d'environ $\pm 0,9 \log \mathrm{UFC} / \mathrm{ml}$; cette précision semble pouvoir être améliorée $( \pm 0,7 \log \mathrm{UFC} / \mathrm{ml})$ lorsqu'on analyse des échantillons de lait de citernes. Quelques hypothèses peuvent être émises pour expliquer cet écart. En effet, les laits de citerne ont généralement fait l'objet d'une conservation prolongée à basse température, entraînant la prolifération des seuls Pseudomonas psychrotrophes (RICHARD, 1981). La meilleure relation entre les deux méthodes peut alors résulter de deux facteurs : une flore relativement homogène et des cellules bactériennes en phase exponentielle de croissance. A l'inverse, les échantillons de lait de producteurs proviennent du mélange du lait d'une seule traite qui n'a donc fait l'objet d'aucune conservation; la nature de la flore et son métabolisme varient donc de façon plus importante d'un échantillon à l'autre.

Il ne nous est pas possible de déterminer avec précision la limite de détection de la technique expérimentale, compte tenu de la nature des échantillons faiblement contaminés; en effet, le comportement différent de ces échantillons peut être lié à leur faible niveau de contamination mais également à leur composition chimique et/ou la nature de leur flore. Il semble cependant qu'elle soit inférieure à $10^{4} \mathrm{UFC} / \mathrm{ml}$.

Compte tenu du principe de la technique étudiée, sa justesse est nettement moins satisfaisante que celle des techniques de microscopie en épifluorescence, comme la DEFT ( $\mathrm{s}_{\mathrm{y}, \mathrm{x}}=0,25$ d'après DASEN et al., 1987). Par contre, elle est sensiblement identique à celle d'autres techniques indirectes, comme le dosage de l'ATP par bioluminescence (BossuYT, 1982b ; BUCHET, 1985). Par rapport à cette dernière, les mesures d'impédance présentent quelques avantages : une limite de détection plus faible, de l'ordre de $10^{4} \mathrm{UFC} / \mathrm{ml}$, en raison de l'incorporation d'une phase d'incubation assez longue ; une automatisation complète, avec cependant quelques problèmes de fiabilité que nous avons pu noter au cours de notre expérimentation. En revanche, l'impédance présente les inconvénients d'avoir un temps de réponse plus long et un coût élevé de l'appareillage.

Sur le plan théorique, notre étude souligne l'intérêt d'exprimer la répétabilité d'une technique instrumentale dans la même unité que celle de la technique de référence. En effet, dans le cas de l'ensemencement en surface par exemple, la répétabilité de la technique peut sembler très satisfaisante puisque le coefficient de variation (en heures) est en moyenne de $5,1 \%$; cependant, après conversion en $\mathrm{UFC} / \mathrm{ml}$, ce coefficient atteint $25 \%$ et est donc largement supérieur à celui des techniques classiques de dénombrement. 


\section{Remerciements}

Les auteurs remercient la Société BActomatic pour le prêt de l'appareil et l'aide technique qu'elle leur a apportée.

\section{Références bibliographiques}

ANONYME, 1972. Standard methods for the examination of dairy products. American Public Health Association, 13th ed., New York.

ANONYME, 1985. Milk. Definition and evaluation of the overall accuracy of indirect methods of milk analysis. Application to calibration procedure and quality control in dairy laboratory. Norme Provisoire FIL-IDF 128.

BossuYT R., 1981. Determination of bacteriological quality of raw milk by an ATP assay technique. Milchwissenschaft, 36, 257-260.

Bossuyt R., 1982a. Techniques modernes d'appréciation de la qualité bactériologique du lait. Tech. Lait., (969), 9-19.

BossuYt R., 1982b. A 5 - minute ATP platform test for judging the bacteriological quality of raw milk. Neth. Milk Dairy J., 36, 355-364.

Bottazzi V., Dellaglio F., Sarra P.G., 1972. Creaming of milk and bacterial agglutination of microorganisms. IV Mechanism causing fat and bacterial agglutination. Sci. Tec. Latt. Casearia, $23,287-304$.

Buchet C., 1985. Appréciation rapide de la qualité bactériologique du lait grâce au dosage de l'ATP par bioluminescence. DEA Science et Technologie du Lait, Faculté des Sciences de Caen.

Cady P., Hardy D., Martins S., Dufour S.W., Kraeger S.J., 1978. Automated impedance measurements for rapid screening of milk microbial content. J. Food Prot., 41, 277-283.

CAMPBell J.E., Gilchrist J.E., 1973. Spiral plate technique for counting bacteria in milk and other foods. Dev. Ind. Microbiol., 14, 95-102.

Dagnelie P., 1970. Théorie et méthodes statistiques. Volume 2. Les méthodes de l'inférence statistique. Editions J. Duculot SA, Gembloux.

Dasen A., Piton C., Grappin R., Guerry P., 1987. Évaluation de la technique DEFT associée à un comptage visuel ou à un comptage par analyseur d'image pour la numération de la flore totale du lait cru. Lait, 67, 77-95.

Donnelly C.B., Gilchrist J.E., Peeler J.T., Campbell J.E., 1976. Spiral plate count method for the examination of raw and pasteurized milk. Appl. Environ. Microbiol., 32, 21-27.

FirstenBERG-EDEN R,, 1984. Collaborative study of the impedance method for examining raw milk samples. J. Food Prot., 47, 407-412.

FirstenberG-Eden R., Eden G., 1984. Impedance Microbiology. Research Studies Press LTD, John Wiley \& Sons INC, Chichester, England.

Firstenberg-Eden R., Tricarico M., 1983. Impedimetric determination of total, mesophilic and psychrotrophic counts in raw milk. J. Food Sci., 48, 1750-1754.

FirSTENBERG-Eden R., Zindulis J., 1984. Electrochemical changes in media due to microbial growth. J. Microbiol. Methods, 2, 103-115.

Gilchrist J.E., Campbell J.E., Donnelly C.B., Peeler J.T., Delaney J.M., 1973. Spiral plate method for bacterial determination. Appl. Microbiol., 25, 244-252.

GNAN S., LuedECKE L.O., 1982. Impedance measurements in raw milk as an alternative to the Standard Plate Count. J. Food Prot., 45, 4-7.

HADLEY W.K., SENYK G., 1975. Early detection of microbial metabolism and growth by measurement of electrical impedance. In : Microbiology, American Society for Microbiology, Washington, DC, $12-21$. 
JARviS B., LACH V.H., WOOD J.M., 1977. Evaluation of the spiral plate maker for the enumeration of microorganisms in foods. J. Appl. Bacteriol., 43, 149-157.

NiEUWENHOF F.F.J., HoOlWERF J.D., 1987. Impedance measurement as an alternative to the plate count method for estimating the total count of bacteria in raw milk. J. Food Prot., 50, 665-668.

O'CONNOR F., 1979. An impedance method for the determination of bacteriological quality of raw milk. Ir. J. Food Sci. Technol., 3, 93-100.

O'CONNOR F., 1984. Rapid test methods for assessing microbiological quality of milk. Aust. J. Dairy Technol., 39, 61-65.

O'TOOLE D.K., 1983. A review : methods for the direct and indirect assessment of the bacterial content of milk. J. Appl. Bacteriol., 55, 187-201,

Peeler J.T., Gilchrist J.E., Donnelly C.B., Campbell J.E., 1977. A collaborative study of the spiral plate method for examining milk samples. J. Food Prot., 40, 462-464.

Pettipher G.L., 1981. Rapid methods for assessing bacterial numbers in milk. Dairy Ind. Int., 46, $15-23$.

RiCHARD J., 1980. Influence de l'agitation du lait cru sur les résultats de dénombrement de sa flore totale à l'aide d'une anse calibrée. Lait, 60, 211-225.

RiCHARD J., 1981. Influence de diverses méthodes de nettoyage des machines à traire sur la " qualité de conservation 》 du lait cru à basse température. Lait, 61, 354-369.

Richard J., Krebs C., Walter J., Grappin R., 1981. Comparaison de divers modes d'agitation des échantillons de lait cru en vue de la numération de sa flore totale suivant la méthode Thompson. Lait, 61, 250-260.

Suhren G., HeEsChen W., 1985. Zur Impedanz als Messgrösse für die bakteriologische Untersuchung von Milch und Milchprodukten. Dtsch. Molk. Ztg., 51/52, 1710-1717.

SuHRen G., HeEsChen W., 1987. Impedance assays and the bacteriological testing of milk and milk products. Milchwissenschaft, 42, 619-627.

Thomas S.B., Hobson P.M., Bird E.R., King K.P., Druce R.G., Cox D.R., 1962. The microflora of raw milk as determined by plating on yeastrel milk agar incubated at $20^{\circ} \mathrm{C}$. J. Appl. Bacteriol., 25, 107-115.

Twomey A., Crawley W.E., 1968. The microflora of raw milk. N.Z. J. Dairy Technol., 3, 44-48. 